\section{A case of Pulmonary Tuberculosis complicated by Pulmonary Thromboembolism}

\author{
Jiawen Wang, Changwu Wan, Yuanhe Wang, Mingjie Li, \\ Le Chen and Wang Jie*
}

Department of Forensic Medicine, Guizhou Medical University, Guizhou, China

\section{Abstract}

We report a case of pulmonary embolism complicated with pulmonary tuberculosis. A 48-year-old woman suffered from pulmonary tuberculosis more than 6 years without formal treatment. Recently, she went to hospital because of "chest tightness and dyspnea", and died in the process of admission to hospital. Pulmonary embolism was found by autopsy and histological examination. We analyzed the relationship between pulmonary tuberculosis and pulmonary thromboembolism and the problems we should pay attention to in forensic pathology.

\section{More Information}

*Address for Correspondence: Wang Jie, Department of Forensic Medicine, Guizhou Medical University, Guizhou, China, Tel: 13027817068; Email: wjwwfs@126.com; wj6400@gmc.edu.cn

Submitted: 19 February 2020

Approved: 10 March 2020

Published: 14 March 2020

How to cite this article: Wang J, Wan C, Wang Y, Li M, Chen L, et al. A case of Pulmonary Tuberculosis complicated by Pulmonary Thromboembolism. J Forensic Sci Res. 2020; 4: 004-006.

DOI: 10.29328/journal.jfsr.1001018

ORCiD: orcid.org/0000-0002-9382-9496

Copyright: @ 2020 Wang J, et al. This is an open access article distributed under the Creative Commons Attribution License, which permits unrestricted use, distribution, and reproduction in any medium, provided the original work is properly cited.

Keywords: Pulmonary tuberculosis; Pulmonary artery; Thromboembolism

\section{Introduction}

A 48-year-old female Lee, suffering from "pulmonary tuberculosis" for more than 6 years, has brokenly taken "antituberculosis drug (Rifampin, isoniazid, ethambutol)" against the disease. For nearly a year, her physical activity was significantly reduced because of chronic fatigue. Constantly stayed in bed, sometimes she could handle simple household chores when her condition was good. Recently, accompanied by her family, she was admitted to hospital due to "chest congestion and breathing difficulties". After preadmission CT testing, she fell on the way to handle hospital procedure and later died despite of emergency rescue efforts. As the patient died without being hospitalized, sputum tuberculosis test and blood test have not been done. According to the family, the patient had no history of drug use. She lived in situ for a long time, had not been to the epidemic area, and had no history of smelting. There was no previous history of heart disease, diabetes, cerebrovascular disease, etc had been found for her?

\section{Necropsy}

External examination: Liver mortis could be seen at the unpressed portion of backside and which was dark red. Putrefactive networks appeared on the limbs. There was congestion on face and throat, cyanochroia on the lactulum unguis and an injection needle holes on the left inner ankle. No injection pinhole found in other parts.

Autopsy: No injury could be seen in the head and brain.

\section{A) Check for updates \\ OPEn ACCESS}

The cerebral gyrus enlarged and the sulci narrowed. Chest examination showed that both lungs were swelling, the capsule was dark red, smooth and intact. A small amount of hemorrhagic spots were under the serosa. Congestion appeared on the section and dark red and tenacious cylindrical solid mass that separated from the tube wall existed in the right pulmonary artery branch. Some gray nodules could be seen in the section of right lower lung (Figure 1). There were about $200 \mathrm{ml}$ light yellow liquid in right pleural and no blood or liquid in left pleural. There was no hematocele in pericardial cavity. The heart weighs $350 \mathrm{~g}$. The size and shape of the heart were normal. The thickness of left and right ventricular walls

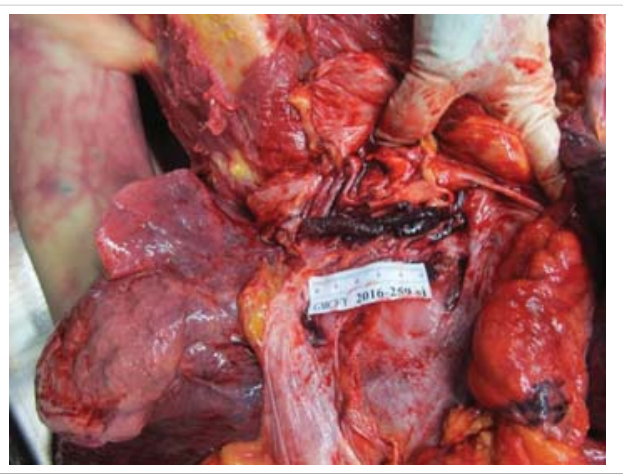

Figure 1: Dark red solid masses could be seen in the pulmonary arteries. 
were $1.3 \mathrm{~cm}$ and $0.3 \mathrm{~cm}$ respectively. No abnormality was found in the diameter of each cardiac valve. The thickness of coronary artery wall is normal and lumen is unobstructed. The surface of liver, bilateral kidneys, spleen and pancreas were dark red. The membrane of the above organs is tight. The weight of the above organs was within the normal range, and no other abnormality was found. No ulcer or tumor was found in the gastric wall and intestinal wall, and no obstruction or stenosis was found in their lumen. No thrombus was found in the deep veins of both lower limbs.

Histopathological examination: The membrane tissues of the lung s loose, and the pulmonary interstitial blood vessel and the alveolus wall capillary telangiectasia dilated and congested. Some tuberculosis nodules could be seen. There were caseous necrosis in the central area, and fibrous cells, epithelioid cells and Langerhans cells were around where. The lymphocytes and fibrous tissue could be seen at the edges. The solid mass in the branch of the right pulmonary artery consists of reddish, unstructured, branched platelet trabeculae and red blood cells filled with trabecular fibrae sanguis meshwork (Figures 2,3). The other organs appear to be congested and (or) edematous.

Expert opinion: Lee is conformed to the death of acute circulatory and respiratory failure caused by Pulmonary Tuberculosis complicated by Pulmonary Thromboembolism.

\section{Discussion}

In forensic pathology, the death case of pulmonary tuberculosis with pulmonary thromboembolism is rare

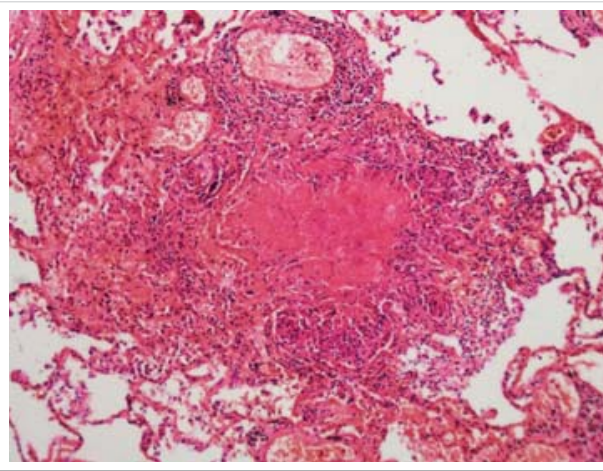

Figure 2: In the lung tissue, there were some caseous necrosises.

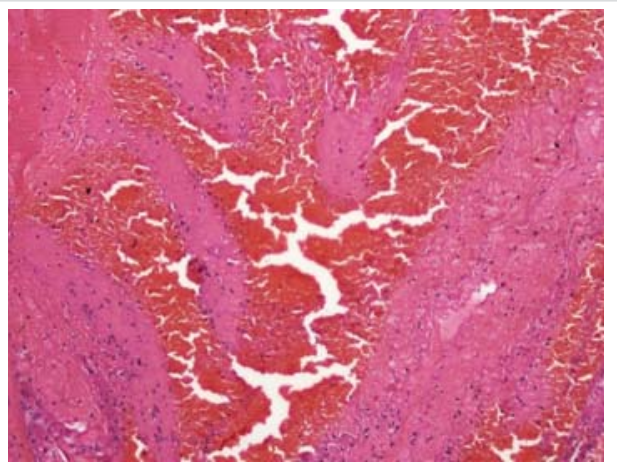

Figure 3: There was a mixed thrombus in the pulmonary artery. in economically developed areas. As an economically underdeveloped province in southwestern China, Guizhou is still frequently encountered in cases of death from tuberculosis. According to clinical statistics, the proportion of pulmonary tuberculosis with pulmonary thromboembolism is approximately $1 \%$ [1]. Thromboembolism is refer to the thrombus fall off and form embolus, the embolus with blood running incarcerated in pulmonary artery that caused the disease which was with pulmonary hypertension and secondly disease of heart failure for the main performance. It is common in patients with lower extremity deep vein thrombosis history. The clinical manifestation often contained refractory hypoxemia, dyspnea, hemoptysis, syncope and other symptoms [2-4]. Venous thrombosis is the most common cause of pulmonary embolism. The majority of pulmonary embolism's primary lesion could be found, only $6 \%$ could not [5]. Long-term fixed position, surgery, trauma, acute inflammation (such as pneumonia) or hormone therapy could cause venous thrombosis, thus the pulmonary embolism could be activated [6-8].

Tuberculosis is a chronic pulmonary infectious disease caused by mycobacterium tuberculosis, and its relapsing and tuberculosis bacillus resistance are the primary factor for cure [9]. The deceased had tuberculosis for more than 6 years and there was no standard system therapy. The double lung surface appeared to swelling in the autopsy, and the chorion was smooth, intact and dark red. A small amount of bleeding point could be seen under the serous membrane. The cut surface was filled with blood. And it could be seen white, hard nodules in the lower right side of the lung. A cylindrical solid mass could be seen in the right pulmonary artery, which was dark red, soft texture and not adhesion to the wall of the blood vessel. The histological examination shows the local lesion nodules. The solid mass in the pulmonary arterial branch was composed of branched platelet trabecular girder and the red blood cells full of fibrin net. The above performances were consistent with the pathological features of pulmonary tuberculosis and pulmonary embolism [10].

The tuberculosis patients without standard system treatment was difficult to be cured. And causing the body chronic consumption, labor and activity limitation. It also could induce the anti-thrombin and protein $\mathrm{C}$ decreased, fibrinogen level increased, platelet aggregation increased and fibrinolytic damage, etc. In this way, the blood of the vein was blocked, high coagulation and eddy current, which could promote or aggravate the formation of venous thrombosis $[11,12]$. When the thrombus falls off, it becomes an embolus. It is easy to block the pulmonary artery which is similar to the caliber of the pulmonary artery, and then cause the symptoms of pulmonary hypertension, secondary respiratory and circulatory failure, leading to dead. It causes symptoms such as pulmonary hypertension and secondary respiratory and circulatory failure leading to death $[1,13]$. 
In this case, the relationship between tuberculosis and drug addiction cannot be avoided. For drug addicts, because the long-term use of syringes causes damage to blood vessels, it is easy to cause venous thrombosis. Thrombus runs with blood flow after falling off, and it is easy to cause embolism when it reaches pulmonary artery. According to the information provided by the family members, the patient had no drug use history. From the perspective of autopsy, no new and old scars of injection pinhole were found in the whole body of the dead, and no thrombosis was found in Lower limbs of the body. Therefore, it can be ruled out that the formation of thrombosis is related to drug injection.

As this patient had no history of trauma, it could be excluded that the pulmonary embolism of deep vein thrombosis was caused by trauma. The limitation of this paper was that venous thrombosis was not found. However, we still believed that the Emboli which plug into the pulmonary artery were formed by the shedding of venous thrombus, and was associated with tuberculosis disease. And it also reminded us that in the treatment of tuberculosis, we should pay attention to the prevention of pulmonary embolism.

\section{Funding}

This work was supported by National Sciences of China (NSFC) [Grant number: 81660309], Teaching Engineering Project of Guizhou Medical University (Key project, grant number: jg201907).

\section{References}

1. Hyeyoung P, Seung-IC, Jae KL. Clinical Characteristics of Coexisting Pulmonary Thromboembolism in Patients with Respiratory Tuberculosis. Am J Med Sci. 2017; 353: 166-171.

PubMed: https://www.ncbi.nlm.nih.gov/pubmed/28183418

2. Razafimanjatoa NN, Vitab OA, Randriamizaob MR. Lethal left cardiac thromboembolism in infant: A rare complication of pulmonary tuberculosis. Respir Med Case Rep. 2019; 26: 98-100. PubMed: https://www.ncbi.nlm.nih.gov/pubmed/30560053

3. Zhou YP. Pulmonary embolism like pulmonary tuberculosis: a case report. Pan Afr Med J. 2017; 22: 191-193.

PubMed: https://www.ncbi.nlm.nih.gov/pubmed/25309662

4. Mohan B, Kashyap A, Whig J. Pulmonary embolism in cases of pulmonary tuberculosis: A unique entity. Indian J Tuberc. 2011; 58: 84-87. PubMed: https://www.ncbi.nlm.nih.gov/pubmed/21644396

5. Dong LX, Chen BY. Multiple filling defects in double pulmonary arteries. Chinese J Tuberculosis and Respiratory. 2007; 10: 793-794.

6. Konstantinides SV, Torbicki A, Agnelli G. 2014 ESC Guidelines on the diagnosisand management of acute pulmonary embolism The Task Force for the Diagnosis and Management of Acute Pulmonary Embolism of the European Society of Cardiology (ESC). Eur Heart J. 2014; 35: 3033-3080.

7. Schmidt M, Horvath PE, Thomsen RW. Acute infections and venous thromboembolism. J Intern Med, 2012; 271: 608-618. PubMed: https://www.ncbi.nlm.nih.gov/pubmed/22026462

8. Smeeth L, Cook C, Thomas S. Risk of deep vein thrombosis and pulmonary embolism after acute infection in a community setting. Lancet. 2006; 367: 1075-1079.

PubMed: https://www.ncbi.nlm.nih.gov/pubmed/16581406

9. Wang FQ, Ni YQ. Pulmonary embolism in patients with pulmonary tuberculosis. Clini Pulmonary J. 2012; 17: 1353-1354.

10. Zhang YB, Tang F, Zhou Q. Investigation of pulmonary thromboembolism in patients with pulmonary tuberculosis and suspected pulmonary tuberculosis. Chinese J Anti-tuberculosis Med. 2008; 30: 473-475.

11. Robson SC, White NW, Aronson I. Acute-phase response and the hypercoagulable state in pulmonary tuberculosis. Br J Haematol. 1996; 93: 943-949.

PubMed: https://www.ncbi.nlm.nih.gov/pubmed/8703831

12. Turken $\mathrm{O}$, Kunter $\mathrm{E}$, Sezer M. Hemostatic changes in active pulmonary tuberculosis. Int J Tuberc Lung Dis. 2002; 6: 927-932. PubMed: https://www.ncbi.nlm.nih.gov/pubmed/12365581

13. Satyasarathi J, Arun G, Swapnil M. Pulmonary Thromboembolism: A Rare Complication of Pulmonary Tuberculosis. J Chest. 2014; 145: 101-102. 